\title{
Search for High-Energy Neutrinos from Populations of Optical Transients
}

\author{
Robert Stein* for the IceCube Collaboration \\ DESY Zeuthen, Platanenallee 6, 15738 Zeuthen, Germany \\ E-mail: robert.steinddesy.de
}

\begin{abstract}
Since the detection of high-energy cosmic neutrinos at the IceCube Neutrino Observatory in 2013, there has been an on-going search to find suitable transient or variable source candidates. Despite recent evidence identifying a flaring blazar as a possible neutrino source, the vast majority of the diffuse neutrino flux measured by IceCube remains unexplained. The latest IceCube results testing time-dependent correlation between neutrinos and Tidal Disruption Events (TDEs) are presented, limiting the contribution of jetted and non-jetted TDEs of the diffuse astrophysical neutrino flux to be less than $1.3 \%$ and $26 \%$ respectively. In addition, a dedicated search for neutrinos from the extraordinary transient AT2018cow are presented, and upper limits on the integrated neutrino emission are derived. Expected improvements from new and upcoming time domain optical surveys (such as ZTF and LSST) are also introduced.
\end{abstract}

The New Era of Multi-Messenger Astrophysics - Asterics2019

25 - 29 March, 2019

Groningen, The Netherlands

${ }^{*}$ Speaker. 


\section{Introduction}

The IceCube Neutrino Observatory is a cubic-kilometer array buried $1.5 \mathrm{~km}$ beneath glacier ice at the geographic south pole [1]. When neutrinos undergo charged-current or neutral-current interactions in the ice, daughter leptons emit Cherenkov light that can be detected by IceCube's 5160 Detector Optical Modules (DOMs). In 2013, IceCube discovered a diffuse flux of highenergy astrophysical neutrinos [2], and there has since been an ongoing search to find potential source candidates. Auto-correlation analyses searching for steady neutrino sources, neutrino flares or coincident neutrino multiplets have so far failed to find any significant clustering within the neutrino flux (e.g [3]). The consistency of this flux with an isotropic distribution suggests that it has a predominantly extragalactic origin.

Neutrino astronomy is generally limited by the overwhelming atmospheric background, but this can be overcome with two complementary approaches. In the neutrino-driven approach, neutrino data is used to search for possible counterparts. Such an approach forms the basis of the IceCube Realtime Program, in which likely astrophysical neutrinos are identified in real-time and immediately distributed as "alerts" to astronomers [4]. One successful example was the followup of IC170922A, a high-energy neutrino that was found in coincidence with a flaring blazar, for which a chance association was disfavoured at the level of $3 \sigma$ [5]. However, because only a handful of neutrinos are identified with these filters each year, it can be hard to make statistically-significant statements about source populations using this approach. These searches are further hampered by the abundance of undetected counterparts that would be expected for most neutrino source populations.

In the alternative source-driven approach, pre-defined catalogues are tested for excesses in neutrinos, with energy-weighting and required spatial coincidence significantly reducing background for searches. Additionally requiring temporal coincidence, either with the lifetime of a transient, or during pre-defined "interesting periods" for variable objects, can further reduce background. Multiple sources can be combined in a stacking analysis, which are designed to detect the sum of many weak individual sources. In all cases, these methods rely on multi-messenger and multiwavelength observations to identify sources to be analysed. Despite IC170922A, previous IceCube analysis has limited the contribution of Fermi-2LAC blazars to the diffuse astrophysical neutrino flux to be less than $30 \%$ [6]. The origin of the vast majority of the neutrino flux thus remains, as yet, undiscovered. Dedicated searches targeting likely sources, including Gamma-Ray Bursts (GRBs), Core-Collapse Supernovae (CCSNe), and starburst galaxies, have so far not revealed any significant excess above background expectations [4]. This motivates the continued analysis of new, untested source classes in an attempt to identify the origin of astrophysical neutrinos.

\section{Tidal Disruption Events}

Within this context, a new analysis was undertaken to search for neutrinos from Tidal Disruption Events (TDEs). A TDE occurs when a star approaches a supermassive black hole (SMBH) on a parabolic orbit [7]. As gravitational acceleration follows a $\frac{1}{r^{2}}$ dependence, the near side of the star will be accelerated more strongly than the far side. The star thus experiences a net tidal force. As the star moves closer to the SMBH, the tidal force increases, until it exceeds the self-gravity 
that holds the star together. At this point, the star is said to be tidally-disrupted, and roughly half of the stellar debris is accreted. In some cases, a relativistic jet can be formed during the accretion process, analogously to a blazar jet. There has been recent theoretical interest in TDEs as potential Ultra-High Energy Cosmic Ray (UHECR) sources, as well as candidate neutrino sources (e.g [8]).

TDEs are a fundamentally rare phenomenon, with rates several orders of magnitude below CCSN rates [9]. However, historically poor detection efficiencies have further exacerbated this, leaving only a handful of reliably-identified TDEs. To date, there have been only 3 on-axis jetted TDEs, and a few dozen candidate non-jetted TDEs [7, 10]. Of these, the majority do not have an unambiguous TDE classification. TDEs themselves are, by their nature, nuclear transients. They can often be confused with flares of Active Galactic Nuclei (AGN), as well as nuclear CCSNe. Due to the greater abundance of these background populations, it can be hard to remove all contamination. Ultimately multiple eras of spectroscopy and photometry are required for a compelling classification. At the time of catalogue compilation for the analysis in October 2017 [10], out of approximately 60 candidate TDEs in the literature overlapping IceCube data-taking, only 13 were judged to be unambiguously classified.

\section{Results}

\subsection{Stacking Search}

The stacking method employed for the analysis did not make assumptions on the relative strength of each tested source, and was thus robust against both catalogue contamination and deviations from a standard-candle neutrino emission scheme [4]. However, in order to meaningfully interpret the results, and extrapolate to constrain emission from the population as a whole, a pure sample is required. Consequently, the non-jetted sample was separated based on robustness of classification, with the "Golden TDEs" being assumed representative of non-jetted TDEs as a whole. The results are shown in Figure 1. Assuming central rate estimates from [9] and [11], we find that non-jetted and jetted TDEs contribute less than $26 \%$ and $1.3 \%$ respectively to the astrophysical neutrino flux. As the contribution from a population is directly proportional to the local population rate, the shaded bands indicate the uncertainty in our limits arising from rate estimates. For TDEs, these rates are the dominant source of uncertainty in neutrino flux. It will require systematic evaluation of observed TDE rates to enable more precise limits on neutrino emission. Any refined rate estimate can be immediately used to directly recalculate limits, without requiring any additional IceCube analysis.

\subsection{AT2018cow}

The discovery of extraordinary transient AT2018cow was a further demonstration of the central importance of multi-messenger observations. This fast, bright, blue transient prompted a comprehensive multi-messenger follow-up campaign, and was variously interpreted as a TDE, an extreme SN or a Magnetar [13]. The observations were consistent with a nearby example of a recentlyidentified population of Fast Blue Optical Transients (FBOTs). A new analysis of AT2018cow, extending from 30 days before peak to 100 days afterwards, did not reveal any significant neutrino emission. The corresponding constraints are illustrated in Figure 2. As before, uncertainty in both classification and rate estimates hinder attempts to constrain neutrino emission from FBOTs. 


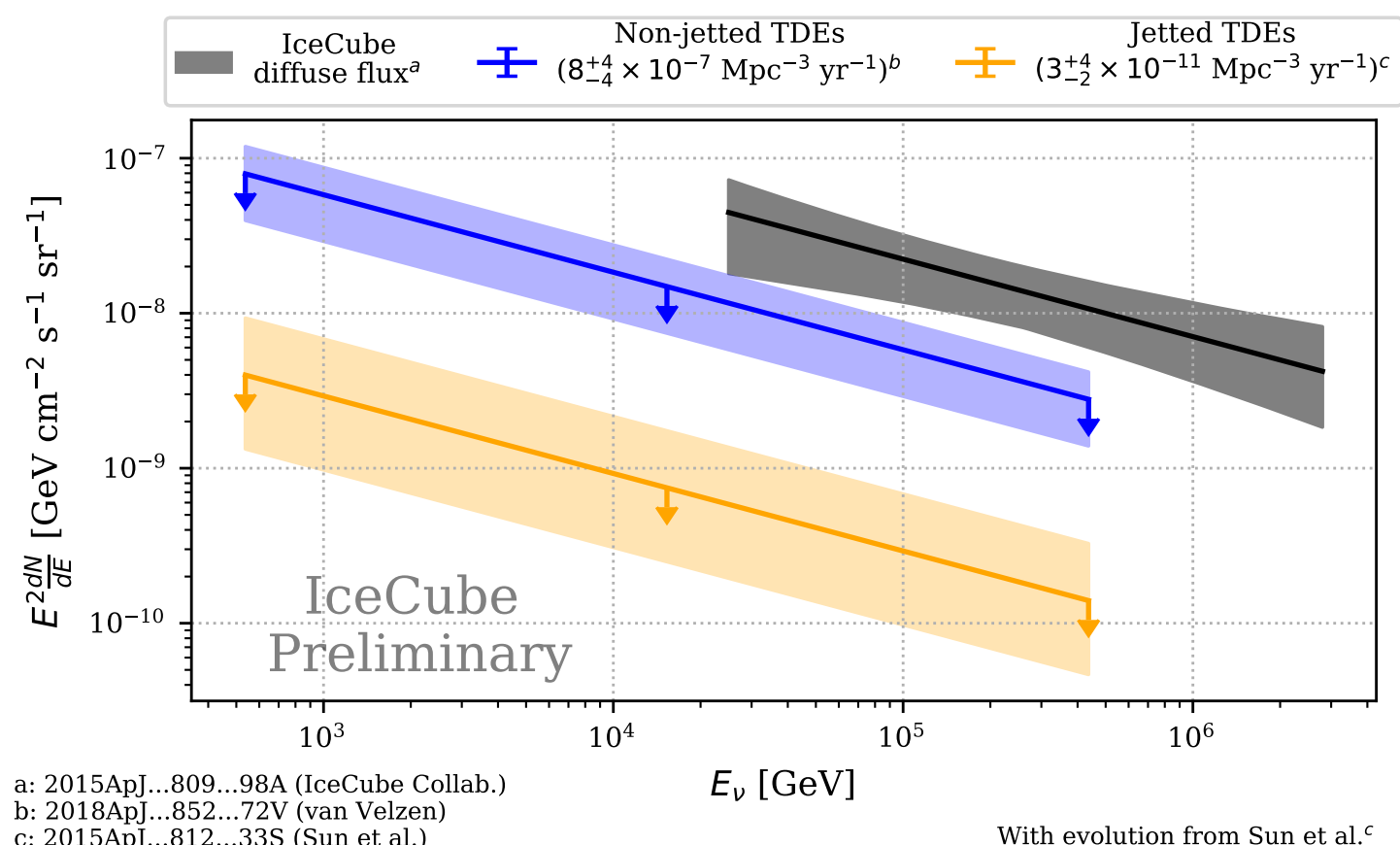

Figure 1: Limits on the contribution of jetted and non-jetted TDEs to the diffuse neutrino flux [12], assuming standard-candle neutrino emission. The shaded bands represent uncertainty in local rate estimates.

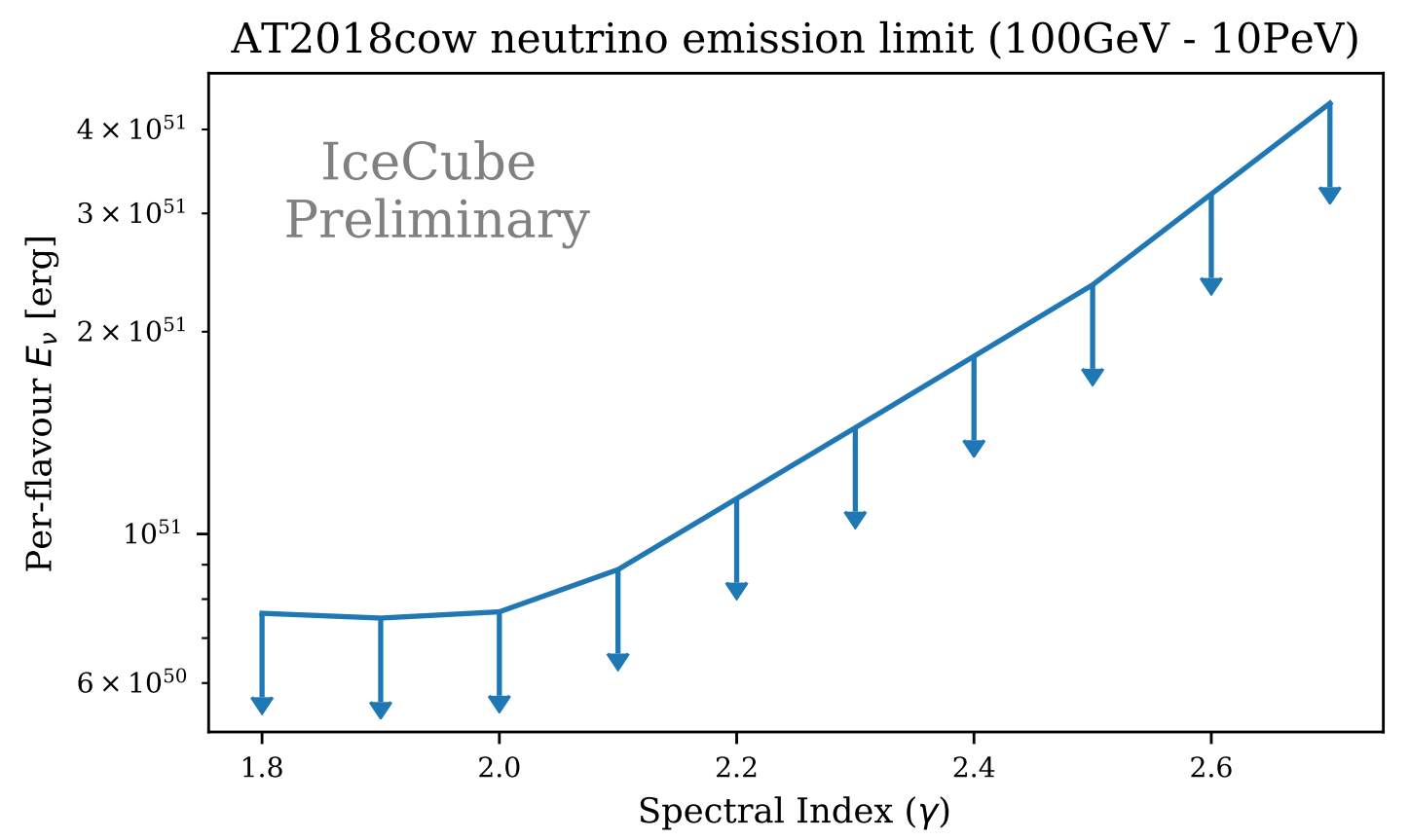

Figure 2: Limits on integrated neutrino emission from AT2018cow as a function of spectral index, assuming a 130 day window from MJD 58256.9 to MJD 58386.9 


\section{Outlook}

The emergence of new telescopes such as ZTF [14], as well as upcoming surveys such as LSST, should aid future analysis. By discovering larger numbers of transients, the sensitivity of searches will grow. Larger samples should also improve rate estimation. Higher cadence observations can greatly reduce background by constraining search windows with greater precision. Consequently, source-driven analysis will continue to grow more powerful.

\section{References}

[1] ICECUBE collaboration, The IceCube Neutrino Observatory: Instrumentation and Online Systems, JINST 12 (2017) P03012 [1612.05093].

[2] ICECubE collaboration, Evidence for High-Energy Extraterrestrial Neutrinos at the IceCube Detector, Science 342 (2013) 1242856 [1311.5238].

[3] ICECUBE collaboration, All-sky Search for Time-integrated Neutrino Emission from Astrophysical Sources with 7 yr of IceCube Data, Astrophys. J. 835 (2017) 151 [1609.04981].

[4] A. J. Stasik, Search for High Energetic Neutrinos from Core Collapse Supernovae using the IceCube Neutrino Telescope, Ph.D. thesis, Humboldt-Universitaet zu Berlin,

Mathematisch-Naturwissenschaftliche Fakultaet, 2018. http://dx.doi.org/10.18452/18729.

[5] ICeCube, Fermi-LAT, MAGIC, AGILE, ASAS-SN, HAWC, H.E.S.S., INTEGRAL, Kanata, Kiso, Kapteyn, Liverpool Telescope, Subaru, Swift NuStar, VERITAS, VLA/17B-403 collaboration, Multimessenger observations of a flaring blazar coincident with high-energy neutrino IceCube-170922A, Science 361 (2018) eaat1378 [1807.08816].

[6] ICECuBE collaboration, The contribution of Fermi-2LAC blazars to the diffuse TeV-PeV neutrino flux, Astrophys. J. 835 (2017) 45 [1611.03874].

[7] S. Komossa, Tidal disruption of stars by supermassive black holes: Status of observations, JHEAp 7 (2015) 148 [1505.01093].

[8] D. Biehl, D. Boncioli, C. Lunardini and W. Winter, Tidally disrupted stars as a possible origin of both cosmic rays and neutrinos at the highest energies, Sci. Rep. 8 (2018) 10828 [1711.03555].

[9] S. van Velzen, On the mass and luminosity functions of tidal disruption flares: rate suppression due to black hole event horizons, Astrophys. J. 852 (2018) 72 [1707. 03458].

[10] K. Auchettl, J. Guillochon and E. Ramirez-Ruiz, New physical insights about Tidal Disruption Events from a comprehensive observational inventory at X-ray wavelengths, Astrophys. J. 838 (2017) 149 [1611.02291].

[11] H. Sun, B. Zhang and Z. Li, Extragalactic High-energy Transients: Event Rate Densities and Luminosity Functions, Astrophys. J. 812 (2015) 33 [1509. 01592].

[12] ICECUBE collaboration, A combined maximum-likelihood analysis of the high-energy astrophysical neutrino flux measured with IceCube, Astrophys. J. 809 (2015) 98 [1507.03991].

[13] D. A. Perley et al., The Fast, Luminous Ultraviolet Transient AT2018cow: Extreme Supernova, or Disruption of a Star by an Intermediate-Mass Black Hole?, Mon. Not. Roy. Astron. Soc. 484 (2019) 1031 [1808.00969].

[14] E. C. Bellm et al., The Zwicky Transient Facility: System Overview, Performance, and First Results, 131 (2019) 018002 [1902.01932]. 